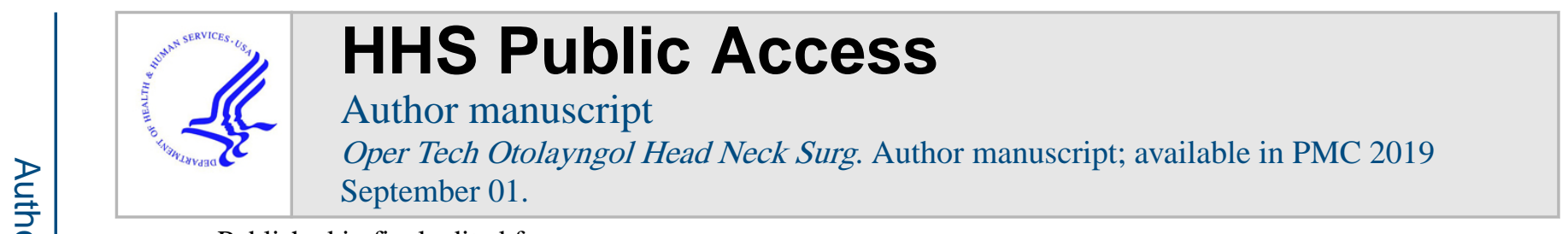

Published in final edited form as:

Oper Tech Otolayngol Head Neck Surg. 2018 September ; 29(3): 157-161. doi:10.1016/j.otot. 2018.06.005.

\title{
Neck dissection for salivary gland malignancies
}

\author{
Serena Byrd, MD and Luc G.T. Morris, MD, MSc, FACS \\ Head and Neck Service, Department of Surgery, Memorial Sloan Kettering Cancer Center, 1275 \\ York Ave, New York, NY 10065
}

\begin{abstract}
Salivary gland carcinomas are diverse, and their biological behavior and surgical management are also variable and somewhat controversial. Cervical lymph node status is an important prognostic variable for salivary gland malignancies. Neck dissection should be undertaken if there is clinical or radiographic evidence of associated nodal metastasis in the neck. However, indications for elective neck dissections in a clinically N0 neck remains a controversial topic. This article describes indications for elective neck dissection in salivary gland malignancies, provides a detailed review of the neck dissection technique, and discusses postoperative management of these patients.
\end{abstract}

\section{Keywords}

Salivary gland malignancy; neck dissection

\section{Background}

Neoplasms of the salivary glands are rare, accounting for approximately 3-6\% of all tumors of the head and neck. The overall incidence is 2.5 to 3.0 cases per 100,000 in the United States. ${ }^{1}$ Although $73 \%$ of the salivary gland neoplasms occur in the parotid gland, only about $15 \%$ are malignant. Submandibular and minor salivary gland neoplasms account for a smaller number of total cases, however, about $40-50 \%$ of these are malignant. ${ }^{2}$ They are classified according to the 2005 World Health Organization, which places salivary gland neoplasms into 24 different histologic subtypes. ${ }^{3}$ Mucoepidermoid carcinoma is the most common malignant salivary gland cancer and is found most commonly in the parotid gland, whereas adenoid cystic carcinomas are more frequently seen in submandibular and minor salivary glands. ${ }^{1}$

\footnotetext{
Corresponding author: Luc G.T. Morris, MD, MSc, FACS, Adlerian Chair, Associate Professor of Surgery, Memorial Sloan Kettering Cancer Center, 1275 York Avenue, New York, NY 10065, Tel 212639 3049, Fax 212717 3278, morrisl@ mskcc.org.

Publisher's Disclaimer: This is a PDF file of an unedited manuscript that has been accepted for publication. As a service to our customers we are providing this early version of the manuscript. The manuscript will undergo copyediting, typesetting, and review of the resulting proof before it is published in its final citable form. Please note that during the production process errors may be discovered which could affect the content, and all legal disclaimers that apply to the journal pertain.

Conflict of Interest: The authors report no proprietary or commercial interest in any product mentioned or concept discussed in this article.
} 
Salivary gland carcinomas (SGC) are diverse and their biological behavior and surgical management are highly dependent on their histological differentiation and grade. Low-grade malignant tumors typically have an indolent course and an overall good prognosis. Highgrade histologic subtypes have an increased risk of regional and distant metastasis and therefore have a worse overall prognosis. Treatment of SGC is primarily surgical with postoperative radiation used in cases such as advanced stage, positive margins, nodal involvement, bone or perineural invasion, and high-grade histology. ${ }^{4,5}$

\section{Indications for neck dissection}

Cervical lymph node status is an important prognostic variable for SGC. Neck dissection should be undertaken if there is clinical or radiographic evidence of associated nodal metastasis in the neck. However, indications for elective neck dissections in a clinically N0 neck remains a controversial topic. Extrapolating from squamous cell carcinoma, if the risk of occult nodal disease is thought to be $>15 \%$ than elective neck dissection has generally been recommended as a staging procedure. ${ }^{5}$ For SDCs, histologic subtype has been shown to significantly predict the rate of occult nodal disease, with incidence highest among salivary duct, high grade mucoepidermoid, undifferentiated, malignant mixed, adenocarcinoma and squamous cell carcinoma $(17-35 \%) .{ }^{7,8}$ Histologic grade has also been found to be a significant predictor of nodal metastasis for high grade mucoepidermoid carcinoma and adenocarcinoma but not for acinic cell carcinoma or other subtypes. ${ }^{7,9}$ Other studies suggest that high-grade tumors, regardless of histologic type, have a significant risk of occult nodal metastases compared to low or intermediate-grade tumors. ${ }^{10}$

It is uncommon for a preoperative workup with fine needle aspiration cytology to be sufficiently specific to confirm a high-grade SGC. If there is clinical suspicion of such an entity, a core needle biopsy may be more informative. Because of these limitations, high-risk histology is often not appreciated at the time of prior surgery, and accordingly, elective nodal dissection is rarely performed. It is important to state that there is no evidence that elective nodal dissection for malignant salivary histologies confers any survival benefit. ${ }^{11}$

Advanced tumor $(\mathrm{T})$ stage is regarded as another independent risk factor for occult nodal disease, with T3 and T4 disease have a much higher rate of regional metastasis than T1/T2 tumors. ${ }^{8} 10,12-13$ Some studies have also suggested that site of primary tumor is a prognostic marker of lymph node involvement in SGC, with lymph node involvement noted in $25 \%$ of parotid gland and $42 \%$ of submandibular gland malignancies. ${ }^{5}$ Parotid tumors with facial paralysis and lymphovascular invasion may also have a higher rate of occult lymph node metastases. ${ }^{14,15}$

Because the above risk factors are generally not appreciated at the time of preoperative workup, the role of elective nodal dissection is limited. It should not be routinely performed for all SGCs. Practically speaking, however, a standard parotidectomy exposes the level IB, II and superior level III neck node compartments. If there is strong intraoperative suspicion of a high-grade malignancy, these nodes can easily be incorporated into the surgical resection. 
Lymph nodes most commonly involved in major SGC are located in levels II and III, suggesting that selective neck dissection levels II-IV is appropriate. ${ }^{9}$ For submandibular carcinoma, selective neck dissection of levels I-III should be performed. In the case of parotid tumors, radiation to the neck has been shown to have similar benefit in regional control as selective neck dissection. If gross regional disease is present, neck dissection followed by radiation should be pursued. ${ }^{16}$ A study by Lim et al showed that patients with intraparotid positive lymph nodes had higher rates of pathologically positive cervical disease in a clinically negative neck, as well as increased rates of locoregional recurrence and worse survival. This suggests that the clinical significance of intraparotid lymph node metastasis can be used as surrogate marker to predict the metastatic potential of parotid cancer, especially in high grade cancers. ${ }^{17}$

Cutaneous malignancies metastatic to the parotid are not primary SGCs but are generally managed with parotidectomy and neck dissection. These diseases include melanoma and squamous cell carcinoma of the skin. When presenting with clinically evident parotid metastases, a selective neck dissection should generally be included with the parotidectomy. The precise extent of complete lymphadenectomy that is required for node-positive melanoma and skin cancers is currently a topic in evolution. These entities are outside the scope of this article, but the technical details of the surgery are quite similar to what would be performed for SGCs.

\section{Surgical Technique}

Perioperative setup and positioning-Before intubation of the patient, it is the responsibility of the surgeon to communicate with the anesthesia team the importance of avoidance of paralysis. A shoulder roll should be placed (or alternatively, the head extended 30 degrees on the bed) to allow for adequate neck extension and exposure. Start by identifying several key landmarks: the angle of mandible, mastoid tip, clavicle, anterior border of the sternocleidomastoid muscle (SCM) and midline. A transverse neck crease is used to camouflage the incision and is carried from the midline to just posterior to the SCM toward the mastoid tip. Local anesthesia is infiltrated into the dermis prior to the setup of the sterile field.

Incision Design-The neck dissection incision is planned in concert with the salivary gland surgery. For carcinomas arising within the parotid glands, minimal extension of the standard modified Blair incision is sufficient to provide access to the neck lymph nodes. Figure 1.

For carcinomas arising within the submandibular gland, a standard mid-neck transverse incision can be used.Figure 2. With a sufficiently long incision, there is no need for curvature of the incision toward the chin or mastoid. Avoidance of vertical incisions in the neck is nearly always cosmetically superior. ${ }^{18}$

Dissection Technique-Subplatysmal flaps are raised superiorly to the angle of the mandible as well as the tail of parotid and inferiorly to the level of the clavicle. The anterior aspect of dissection is the sternohyoid muscle and the posterior limit is the SCM. This approach allows excellent access to levels I-V and can be adjusted according to the lymph 
node levels of interest. Care should be taken to preserve the external jugular vein and greater auricular nerve. These structures will generally eventually require division, but this should be done keeping maximal length should a nerve graft be needed.Fig 3 .

Dissection of level I-If indicated (e.g. tumors arising from the submandibular gland), attention to dissection of level I is first addressed. This usually is similar to a standard level I neck dissection. The fascia overlying the inferior aspect of the submandibular gland is incised. The facial vein is ligated and divided. These structures are elevated off the gland in what is known as the Hayes Martin maneuver, which is helpful in protecting the mandibular marginal nerve. The fascia is elevated to the inferior border of the mandible and its attachments divided. One should be aware of the facial artery and vein in this area, which usually require ligation. Next, free the submandibular gland off the anterior digastric muscle and the underlying lateral aspect of the mylohyoid muscle. Care should be taken to ligate the mylohyoid vessels to avoid bleeding. This allows for the free edge of the mylohyoid muscle to be retracted superiorly and medially. The submandibular gland is retracted inferiorly and blunt dissection of the submandibular fat and lymph nodes is performed, allowing for identification of the lingual nerve and submandibular ganglion and duct. Wharton's duct and the submandibular ganglion are ligated and divided. The contents of the submandibular triangle are dissected in a medial to lateral fashion. The facial artery is identified at the posterolateral aspect of the gland and is divided. The posterior belly of the digastric is identified and the level Ib specimen taken off the digastric. This specimen is kept pedicled to the adjacent level II neck contents. If the contents of level Ia are to be removed, the contralateral anterior belly of the digastric muscle is identified. The superficial fat overlying the anterior bellies of both digastric muscles are removed. This specimen can be removed en bloc or in continuity to level Ib. If removal of perifacial lymph nodes is required, the marginal mandibular nerve is typically identified within the submandibular fascia running just below the angle of the mandible, proximal and superficial to the facial vein. Once identified, the nerve is carefully elevated off the underlying soft tissue to allow for perifacial nodal removal without injury to the marginal mandibular nerve.

In some cases, infiltrative cancers arising from the SMG will require modification of this technique. If necessary, the superior skin flap can be raised in a supra-platysmal plane (rather than subplatysmal) if the tumor is invading the platysma or a suitable margin cannot be achieved otherwise. In rare cases, an ellipse of skin may need to be incorporated at this location. Tumors may also be adherent to the lower border of the mandible, necessitating a marginal resection of bone, or to level I musculature or floor of mouth. In these cases, preservation of the marginal mandibular branch of the facial nerve may not be possible.

Dissection of levels II-IV-The upper subplatysmal flap from the neck is connected with the skin flap over the parotid gland, which is raised just above the parotid fascia. The fascia overlying the sternocleidomastoid (SCM) muscle is divided along is length and is elevated toward the anterior aspect of the SCM. An Allis clamp can be used to retract this fascia medially, while the SCM is retracted laterally. The anterior border of the SCM is opened superiorly to inferiorly using electrocautery. The spinal accessory nerve is identified as it enters the muscle, posterolateral to the jugular vein. This is traced to the inferior border of 
the posterior digastric muscle. Retract the muscle superiorly to visualize CN XI as it crosses over the jugular vein. The superior extent of the dissection of level IIa is inferior to this intersection. Avoid aggressive traction or trauma to $\mathrm{CN} \mathrm{XI}$ as this can result in permanent shoulder dysfunction. If dissection of level IIb is desired, gentle retraction of the accessory nerve inferiorly allows for adequate exposure. It is also possible to remove the level IIb packet separately from the main specimen to avoid unnecessary retraction or compression on the spinal accessory nerve. Electrocautery can be used to remove the fibroadipose tissue from the muscular floor. Once the accessory nerve is freed from the underlying nodal bed, level IIb can be swept under CN XI and left in continuity with level IIa. Next, the cervical rootlets are identified and skeletonized as the fat and fascia are dissected anteriorly toward the jugular vein. This defines the deep plane of the dissection. Care must be taken to preserve the fascia overlying the scalene and levator scapulae muscles to prevent injury to the phrenic nerve and brachial plexus. Medial retraction of the specimen by your assistant is paramount for adequate resection and visualization of important structures. As the dissection is carried anteriorly above the deep cervical fascia, from the posterior border of the SCM (or posterior to SCM if level V is to be addressed) to the carotid sheath. Division of the posterior omohyoid muscle allows for complete exposure of the level IV nodal contents. Care should be taken to avoid injury to the thoracic duct in this area. Because the thoracic duct is difficult to visualized, the contents of level IV should be ligated and tied with a 3-0 silk suture. The neck contents are dissected off the IJV just superficial to the adventitia. Avoid injury to the vagus nerve and carotid artery with this maneuver. The specimen is dissected away from the sternohyoid muscle, branches of the internal jugular vein and external carotid artery. Care should be taken to identify and avoid injury to the hypoglossal nerve which lies deep to the posterior digastric muscle and crosses over the external carotid. The contents of the neck on removed en bloc.

The neck is irrigated with normal saline. A Valsalva is performed to evaluate for adequate hemostasis and a chyle leak. 1-2 Jackson-Pratt drains are placed deep to the SCM. A 3-layer closure is completed: the platysma is re-approximated using 3-0 Vicryl, the subdermal layer with 4-0 vicryl and the skin closed with a 5-0 Monocryl in a subcuticular fashion or Dermabond.

Postoperative Care-Our patients typically remain in the hospital for approximately 1-3 days postoperatively. A neck dressing is not necessary and the neck incision can be left open to air. Bacitracin ointment may be applied to the skin incision but care must be taken not to use this for more than 5 days as skin irritation can result. Consider physical therapy rehabilitation on postoperative day one for shoulder mobilization and strengthening, regardless of the status of the accessory nerve, as some degree of shoulder dysfunction is common. Jackson-Pratt drain(s) are left in place for approximately 3 days and are typically removed thereafter if the output is under $30 \mathrm{~mL}$ in a 24-hour period. Patients are typically discharged on postoperative day 1 with drains, or after drain removal, pending an uncomplicated hospital course.

Postoperative Complications-Postoperative complications are rare following neck dissection but prompt realization and management of the problem is critical to patient care. 
Some more common complications include, but are not limited to: neck infection, hematoma, thoracic duct injury resulting in chyle leak, and neurogenic injury such as marginal mandibular, spinal accessory nerve, phrenic nerve, vagal nerve, cervical plexus, sympathetic chain injury and/or greater auricular nerve.

\section{References:}

1. Shah Jatin, Patel SG, Singh B. Jatin Shah's Head and Neck Surgery and Oncology, Fourth Edition. Philadelphia: Elsevier 2007

2. Eveson JW, Cawson RA. Salivary gland tumours. A review of 2410 cases with particular reference to histological types, site, age and sex distribution. J Pathol 1985; 146: pp. 51-58. [PubMed: 4009321]

3. Barnes L, Eveson JW, Reichart P, Sidransky D, eds Pathology and Genetics of Head and Neck Tumours Kleihues P, Sobin LH, series eds. World Health Organization Classification of Tumours. Lyon, France: IARC Press, 2005 (Paperback, ISBN:9283224175,\$110, www.iarc.fr).

4. Wang X, Luo Y, Li M, Yan H, Sun M, Fan T. Management of salivary gland carcinomas - a review. Oncotarget. 2017; 8(3): 3946-3956. [PubMed: 27992367]

5. Terhaard CH, Lubsen H, Rasch CR, Levendag PC, Kaanders HH, Tjho-Heslinga RE et al. The role of radiotherapy in the treatment of malignant salivary gland tumors. Int J Radiat Oncol Biol Phys. 2005; 61: 103-11. [PubMed: 15629600]

6. Vander PV, Hunt J, Bradley PJ, Haigentz M, Rinaldo A, Mendenhall WM et al. Recent trends in the management of minor salivary gland carcinoma. Head Neck. 2014; 36: 444-55. [PubMed: 23559518]

7. Lau VH, Aouad R, Farwell DG, Donald PJ, Chen AM.Patterns of nodal involvement for clinically N0 salivary gland carcinoma: refining the role of elective neck irradiation. Head Neck. 2014; 36: 1435-9. [PubMed: 24038533]

8. Regis De Brito Santos I, Kowalski LP, Cavalcante De Araujo V, Flavia Logullo A, Magrin J. Multivariate analysis of risk factors for neck metastases in surgically treated parotid carcinomas. Arch Otolaryngol Head Neck Surg 2001; 127: 56-60. [PubMed: 11177015]

9. Lloyd S, Yu JB, Ross DA, Wilson LD, Decker RH.A prognostic index for predicting lymph node metastasis in minor salivary gland cancer.Int J Radiat Oncol Biol Phys. 2010; 76: 169-75. [PubMed: 19386433]

10. Armstrong JG, Harrison LB, Thaler HT, Friedlander-Klar H, Fass DE, Zelefsky MJ et al. The indications for elective treatment of the neck in cancer of the major salivary glands.Cancer. 1992; 69: 615-9. [PubMed: 1730113]

11. Valstar MH, Van den Brekel M, Smeele LF.Interpretation of treatment outcome in the clinically node-negative neck in primary parotid carcinoma: A systematic review of the literature. Head Neck 2010; 32 (10): 1402-11. [PubMed: 20029981]

12. Stennert E, Kisner D, Jungehuelsing M, Guntinas-Lichius O, Schroder U, Eckel HE et al. High incidence of lymph node metastasis in major salivary gland cancer. Arch Otolaryngol Head Neck Surg 2003; 129: 720-3. [PubMed: 12874071]

13. Spiro RH, Huvos AG. Stage means more that grade in adenoid cystic carcinoma. Am J Surg 1992; 164: 623-8. [PubMed: 1334380]

14. Frankenthaler RA, Byers RM, Luna MA, Callender DL, Wolf P, Goepfert H.Predicting occult lymph node metastasis in parotid cancer. Arch Otolaryngol Head Neck Surg. 1993; 119: 517-20. [PubMed: 8484940]

15. Yoo SH, Roh JL, Kim SO, Cho KJ, Choi SH, Nam SY, Kim SY. Patterns and Treatment of Neck Metastases in Patietns with Salivary Gland Cancers.J Surg Oncol. 2015; 111: 1000-6. [PubMed: 25976866]

16. International Head and Neck Scientific Group. Cervical lymph node metastasis in adenoid cystic carcinoma of the major salivary glands. J Laryngol Otol. 2017; 131: 96-105. [PubMed: 27974071]

17. Lim CM, Gilbert MR, Johnson JT, Kim S. Clinical significance of intraparotid lymph node metastasis in primary parotid cancer. Head Neck. 2013; 36: 1634-37 [PubMed: 24123567] 
18. Myssiorek D, Becker GD. Extended single transverse neck incision for composite resections: Does it work? J. Surg. Oncol 1991; 48(2): 101-105. [PubMed: 1921394] 


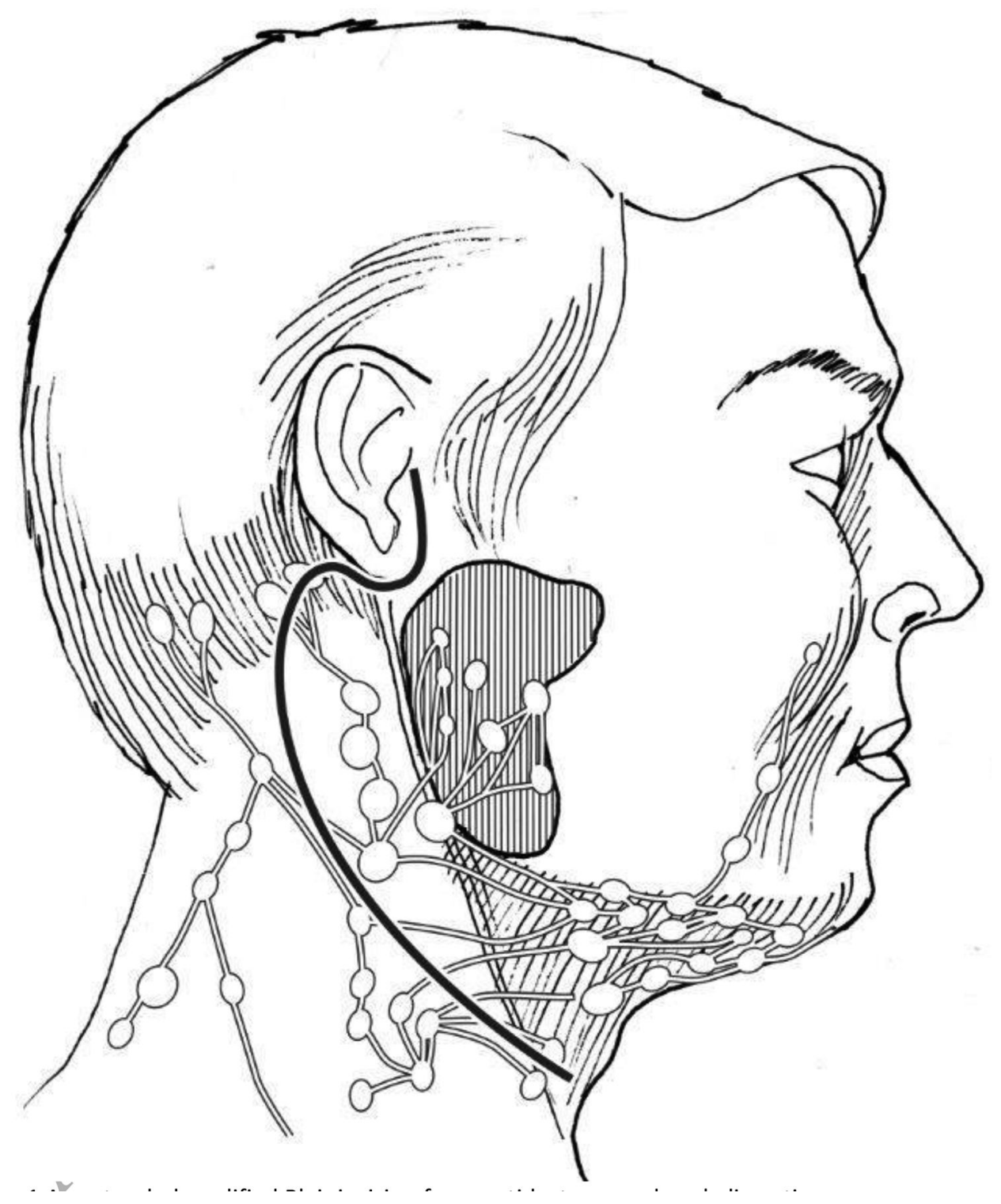

Fig 1.

An extended modified Blair incision for parotidectomy and neck dissection 


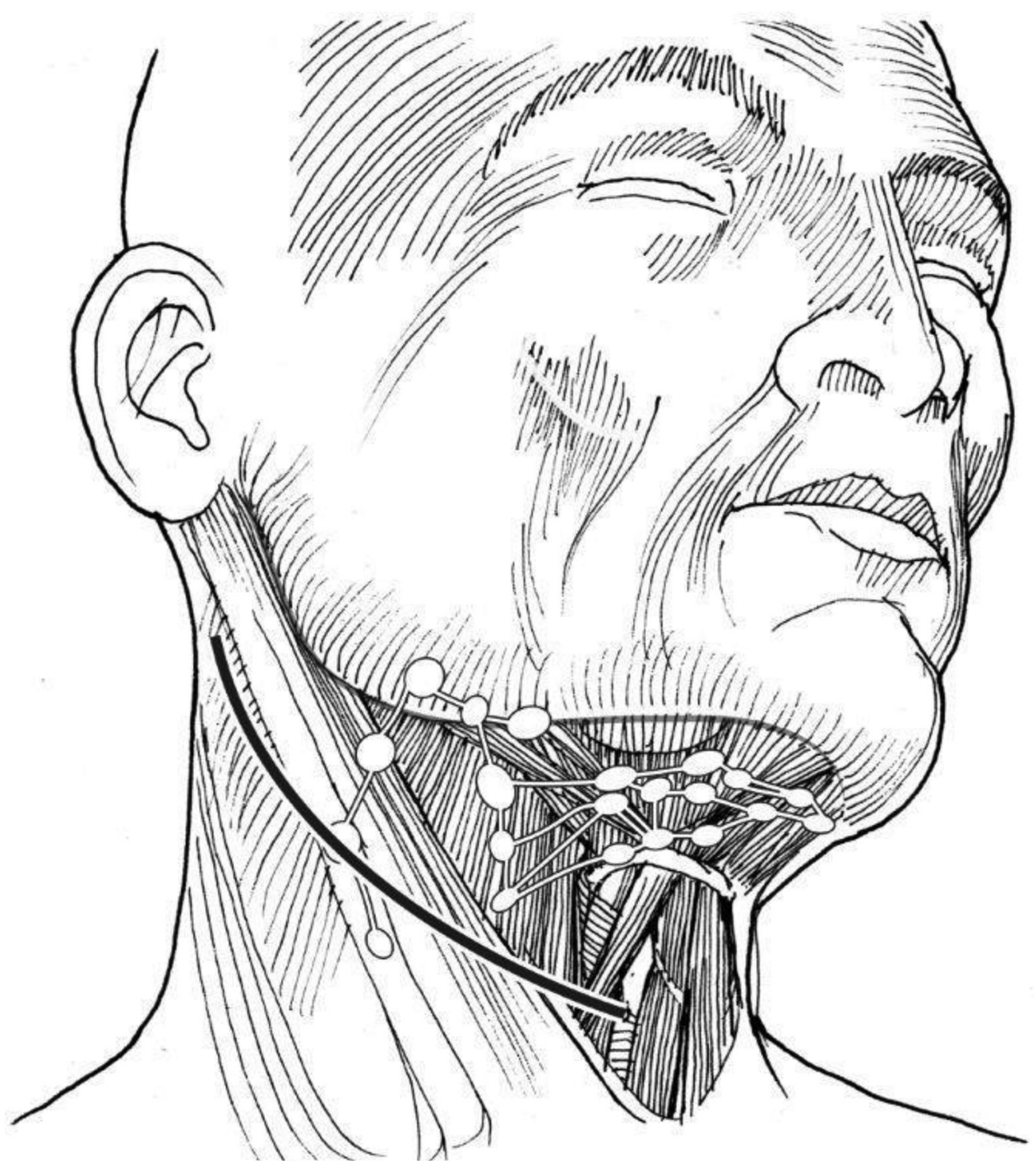

Fig 2.

Transverse incision for submandibular gland resection and neck dissection 


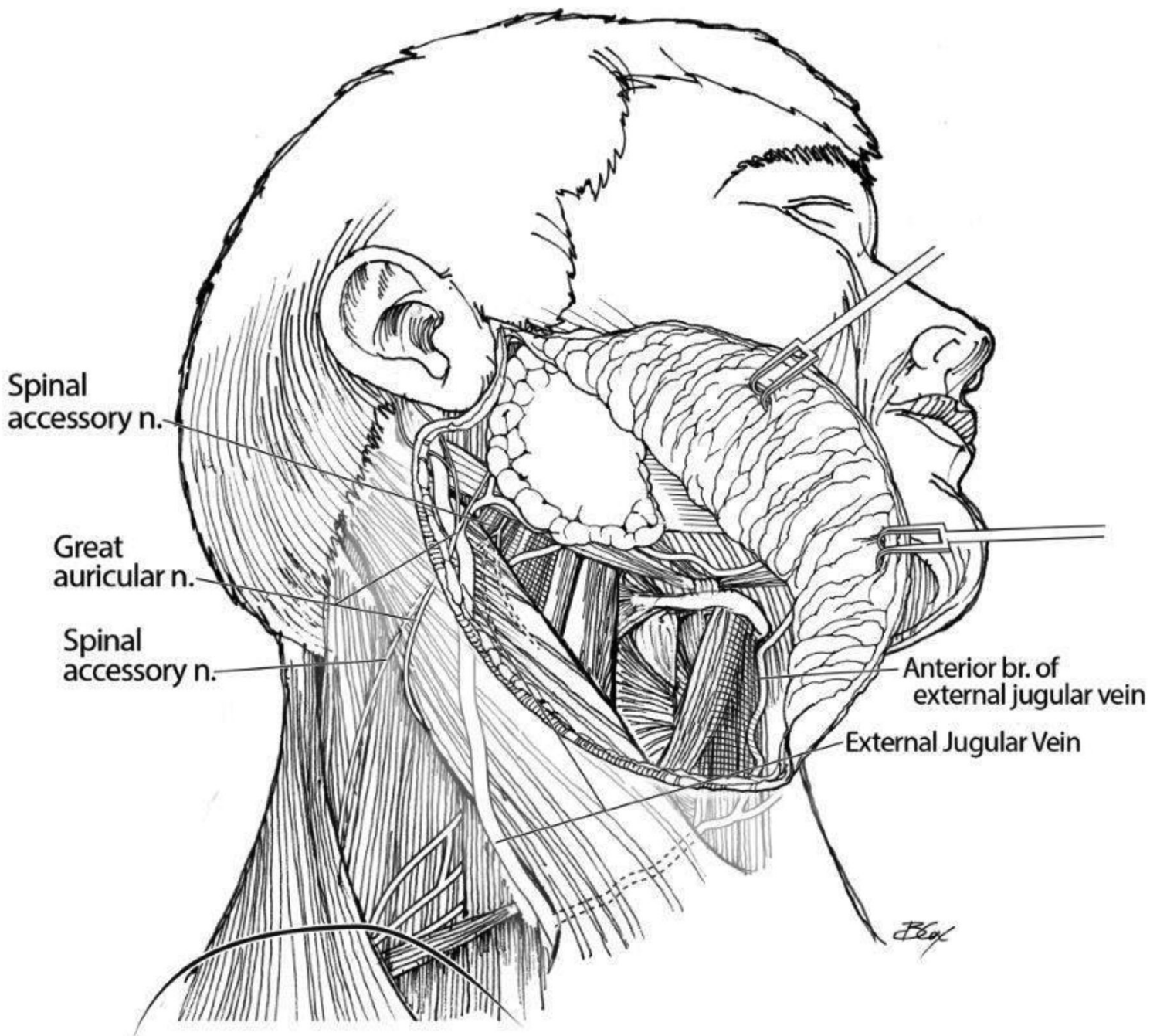

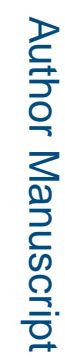




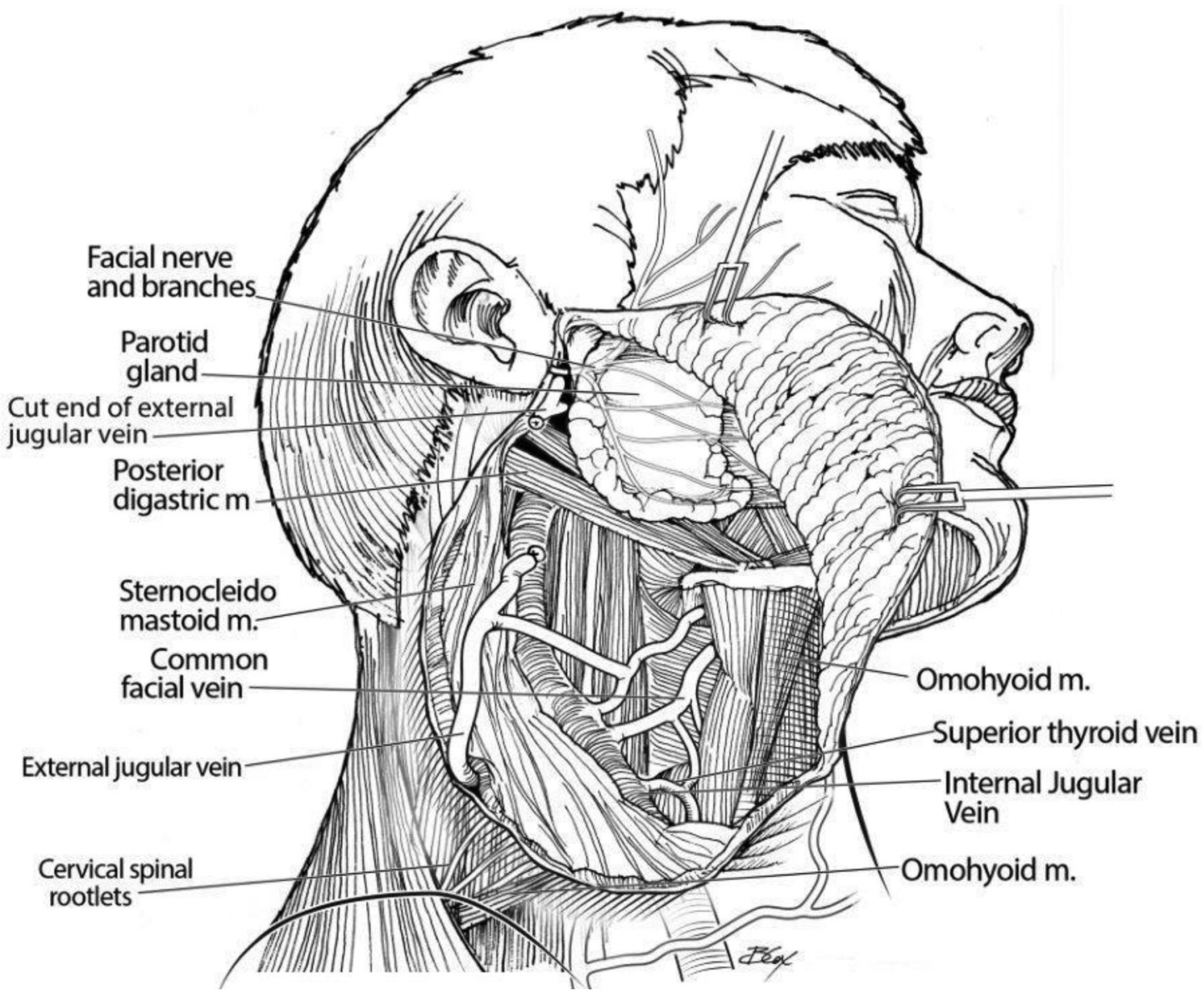

Fig 3.

Fig 3A Elevation of skin flap off of the parotid fascia in continuity with the subplatysmal cervical flap; 3B: Anatomic structures encountered within combined parotid and neck dissection 